Contents available at www.medrech.com

\title{
PEDIATRIC MULTIPLE SCLEROSIS - A CASE REPORT FROM ALBANIA
}

Armand Shehu ${ }^{1}$, Ervin Toçi ${ }^{1}$

1. University Hospital Center "Mother Theresa", Tirana, Albania

\begin{tabular}{l|l} 
ARTICLE INFO & ABSTRACT \\
\hline $\begin{array}{l}\text { Article History } \\
\text { Received: January 2021 } \\
\text { Accepted: March 2021 } \\
\text { Keywords: Albania, acute } \\
\text { disseminated } \\
\text { encephalomyelitis, } \\
\text { childhood, multiple }\end{array}$ & $\begin{array}{l}\text { Multiple sclerosis (MS) is one of the most debilitating diseases with a } \\
\text { considerable financial burden and deteriorating effect on the quality of } \\
\text { life for the affected individual, the family and the entire society. } \\
\text { Although MS affects young adults, it can develop in children as well. In } \\
\text { these cases, the diagnosis of MS could be rather challenging but it } \\
\text { should be timely done in order to start the appropriate treatment and } \\
\text { avoid unfavorable outcomes. We present the case of multiple sclerosis } \\
\text { in a 13-year-old boy in Albania who initially was suspected to be } \\
\text { affected by acute disseminated encephalomyelitis and describe the } \\
\text { diagnosis procedure challenges. }\end{array}$ \\
$\begin{array}{l}\text { Corresponding author* } \\
\text { Armand Shehu }\end{array}$ &
\end{tabular}

O2021, www.medrech.com

\section{INTRODUCTION}

According to the National Multiple Sclerosis Society, Multiple Sclerosis (MS) is "an unpredictable disease of the central nervous system that disrupts the flow of information within the brain, and between the brain and body"

(1). The disease is an inflammatory demyelinating neuro-degenerative disorder of the central nervous system, with immunemediated elements $(2,3)$. Typically, MS is diagnosed in individuals aged between 20 and 40 years old (4). When MS appears before the age of 16, it is called pediatric MS (5). Pediatric MS cases comprise between 2.2\% (6) to $10 \%$ (5) of all MS patients. In some cases, the disease can appear in a fulminant form with severe and frequent relapses associated with significant morbidity and mortality and therefore prompt diagnosis and treatments is indispensable (7). In this context, we describe the case of a 13-year-old boy with multiple sclerosis initially suspected to be affected by acute disseminated encephalomyelitis (ADEM), in Albania.

\section{CASE REPORT}

A 13-year old boy was hospitalized in mid-February 2020 in the neuropediatric clinic in the premises of University Hospital Center "Mother Theresa", Tirana, Albania, with the diagnosis: hemiparesis dexter; febrile condition.

At the time of hospitalization, the child was unable to move and raise his right hand and foot, he was experiencing numbness and tingling in the right-side extremities, difficulty walking, and body temperature of up to 38.5 degrees. 
Medical history: According to the relatives, the child had been suffering from fever for two days and in the morning he was hospitalized he was experiencing numbness and tingling in the right-side extremities, unable to raise and move his right hand and foot, fatigue, difficulty walking (ataxia) and headache; this situation lasted for about two hours and then the child calmed down showing no symptoms at all. After being admitted to the ward, routine tests were carried out as well as CT scan of the head and column, which turned out normal. The next day, the child experienced a relapse of the previous-day episode, showing the same symptoms but that lasted a little bit shorter; since there was a recurrence of symptoms it was suggested to perform the MRI imaging of the head which resulted in lesions of the white matter that were more in favor of a potential acute disseminated encephalomyelitis (ADEM) diagnosis. However, the clinical findings were not supportive of this diagnosis but rather suggested the diagnosis of multiple sclerosis despite the young age of the patient. For this reason some more specific examinations and tests were carried out, including hemogram (WBC 4.200; RBC 4.970.000; HGB 14.1g/dl; HCT 43.0\%; MCV 87 um; PLT 235x 103; SED $16 \mathrm{~mm} / \mathrm{h})$ and biochemical tests [Glycaemia - $80 \mathrm{mg} / \mathrm{dL}$; Azotemia - $32 \mathrm{mg} / \mathrm{dL}$; Creatinine - $0.6 \mathrm{mg} / \mathrm{dL}$; AST -29 u/L; ALT $16 \mathrm{u} / \mathrm{L}$; Bilirubin - $0.4 \mathrm{mg} / \mathrm{dL}$; Total protein $7.7 \mathrm{mg} / \mathrm{dL}$; Sodium - 138m mol/1; Potassium $3.9 \mathrm{~m} \mathrm{~mol} / \mathrm{l}$; Chlorine - $104.8 \mathrm{~m} \mathrm{~mol} / \mathrm{l}$; Fibrinogen - $260 \mathrm{mg} / \mathrm{dl}$; PCR - $0.08 \mathrm{mg} / \mathrm{dl} ;$; Protein electrophoresis - normal; immunophenotype - normal; Vitamin D3 level -12.45 (insufficiency); EBV IgG - 28.57 (positive); EBV IgM - 0.03 (negative)]. In summary, the laboratory examinations revealed a Vitamin D3 deficiency and a positive Epstein-Barr Virus serology.

Life history: The child patient was the second child in a row. Pregnancy was normal. The boy was born via section cesarean with a birth weight of 3150 grams. The patient was breastfed for 9 months and was vaccinated regularly. He had been treated for four years by a pneumo-allergist for asthma with fluticasone, salbutamol and loratadine.

Family history: Nothing important to be noted.

Objective and neurological examination: The child's actual condition was currently good. He reacted and oriented himself according to his biological age. He answered very well to the questions that were being asked, he was coherent for the situation in which he found himself. The boy complained of mild headache in the occipital area. Complained of slight fatigue on the rightside extremities but there was no motor deficit and no ataxia when walking. Deep tendon reflexes were present in both extremities. There was control over sphincters, body and extremities sensitivity was preserved. Urination and defecation were normal.

$E E G$ : Diffuse paroxysmal anomalies.

CT scan of head and column: Normal.

MRI of head: Hyper intense lesions in $\mathrm{T} 2$ of periventricular white matter, corpus callosum splenium. Lesions present mild signal restriction in DWI. Conclusion: Multiple lesions of periventricular white matter probably from acute disseminated encephalomyelitis (ADEM) or metabolic nature (hypoglycemia).

Fundus oculi examination: Normal.

Final diagnosis: After consulting with a team of specialists and based on clinical findings, MRI imaging and biochemical examinations, the final medical diagnosis was Multiple Sclerosis.

Treatment: Based on the clinical, laboratory and imaging results, the child was started on methylprednisolone high dose pulse therapy, valproate and vitamin D3.

With the start of therapy and until its completion the child showed a significant improvement in terms of symptoms which did not reappear anymore. The child underwent 
another cycle of treatment with methylprednisolone after one month and the next head MRI carried out in April did not show any of the previous lesions. The child is on immunomodulatory therapy with interferon beta 1a.

\section{DISCUSSION}

Multiple sclerosis is one of the most debilitating diseases being responsible for a considerable financial burden and a reducing of the quality of life for the affected individuals, their families, caregivers and the entire society $(8,9)$.

Even though it usually affects young adults, it can also appear in children in various forms, including the fulminant ones. For example, research shows that in about $7 \%$ of MS patients there are radiographic features of fulminant disease (10). In child patients that present with fever, headache, meningeal signs and hemiparesis, coupled with CT scan findings, the acute disseminated encephalomyelitis (ADEM) could be first suspected; however, in MS cases, the relapsing-remitting nature of disease might not support such conclusion and therefore there is needed for differential diagnosis with multiple sclerosis in order to set the diagnosis (11). It is clear that diagnosing $\mathrm{MS}$ in pediatric age patients remains challenging due to not welldefined diagnostic criteria and their overlap with ADEM (12). A MRI of the head revealing white matter lesions, in addition to the clinical features, might serve as a basis for the definitive diagnosis of MS (11). This was exactly the case with our young patient who was first suspected to be suffering from ADME but later on was diagnosed with MS.

MS has an inflammatory autoimmune component $(13,14)$. It has been reported that the rate of asthma, type 1 diabetes mellitus and other autoimmune diseases is higher among MS patients (15) whereas other studies have found that MS patients are significantly more likely to have asthma which began before the age of onset of MS symptoms compared to controls without MS (16). Similarly, our patient had an history of asthma in his early childhood, which is another argument in favor of the MS diagnosis in this case.

Our patient was experiencing low levels of Vitamin D. Numerous studies have reported that exposure to sunlight, time spent outside and outdoor activities, especially during childhood, all of which are associated with higher levels of blood Vitamin D, might have a significant protective role against multiple sclerosis $(17,18)$. A recent literature review reported that there seems to be an increasing evidence about the inverse association between the levels of Vitamin D and the risk of developing MS but, nevertheless, the effect of Vitamin D supplements on MS activity remains largely under investigated (19).

Pediatric onset MS is often associated with slower progression compared to adult onset MS, but its associated disability could occur at a younger age and may be less favorable in the non-whites (12). MS patients eventually will experience progressive disability that will have a deep impact on them and their beloved ones (20).

Even though there is no definitive cure for the MS, the diagnosis has to be determined as early as possible in order to start the right treatment as it is shown effective in slowing the disability and prevent unfavorable outcomes associated with disease progression (21). Pediatric medial staff should be aware of the presence of multiple sclerosis and dismiss it even in very young patients and be ready to overcome pediatric MS diagnostic challenges.

CONFLICT OF INTEREST: None declared. REFERENCES

1. National Multiple Sclerosis Society. What is MS. Available at: https://www.nationalmssociety.org/What -is-MS. Last accessed: June 2020.

2. Wootla B, Eriguchi M, Rodriguez M. Is multiple sclerosis an autoimmune 
disease? Autoimmune Dis. 2012; 2012:969657.

3. Martin R, Sospedra M, Rosito M, Engelhardt B. Current multiple sclerosis treatments have improved our understanding of MS autoimmune pathogenesis. Eur J Immunol. 2016;46(9):2078-2090.

4. Tillery EE, Clements JN, Howard Z. What's new in multiple sclerosis? Ment Health Clin. 2018;7(5):213-220.

5. Alroughani R, Boyko A. Pediatric multiple sclerosis: a review. BMC Neurol. $2018 ; 18(1)$ : 27.

6. de Albuquerque RC, de Paula RS, Brito MM, Filho JR, Meguins LC. Pediatric multiple sclerosis-a challenging demyelinating disease: case report and brief review of the literature. Case Rep Pediatr. 2012; 2012:684064.

7. Fernando KTM, James M. Early predictors of rapidly evolving multiple sclerosis: A case report. J Neuroimmunol. 2017; 307:42-46.

8. Maroney M, Hunter SF. Implications for multiple sclerosis in the era of the Affordable Care Act: a clinical overview. Am J Manag Care. 2014;20(11 Suppl): S220-S227.

9. Svendsen B, Grytten N, B $\varnothing$ L, Aarseth $\mathrm{H}$, Smedal T, Myhr KM. The economic impact of multiple sclerosis to the patients and their families in Norway. Eur J Health Econ. 2018;19(9):12431257.

10. Rahmlow MR, Kantarci O. Fulminant demyelinating Neurohospitalist. 2013;3(2):81-91.

11. Lee YJ. Acute disseminated encephalomyelitis in children: differential diagnosis from multiple sclerosis on the basis of clinical course. Korean J Pediatr. 2011;54(6):234-240.

12. Chabas D, Strober J, Waubant E. Pediatric multiple sclerosis. Curr Neurol Neurosci Rep. 2008;8(5):434-441.
13. Yeshokumar AK, Narula S, Banwell B. Pediatric multiple sclerosis. Curr Opin Neurol. 2017;30(3):216-221.

14. Batur-Caglayan HZ, Irkec C, YildirimCapraz I, Atalay-Akyurek N, Dumlu S. A case of multiple sclerosis and celiac disease. Case Rep Neurol Med. 2013; 2013:576921.

15. Edwards LJ, Constantinescu CS. A prospective study of conditions associated with multiple sclerosis in a cohort of 658 consecutive outpatients attending a multiple sclerosis clinic. Mult Scler. 2004;10(5):575-581.

16. Ponsonby AL, Dwyer T, van der Mei I, et al. Asthma onset prior to multiple sclerosis and the contribution of sibling exposure in early life. Clin Exp Immunol. 2006 ;146(3) :463-470.

17. Bjørnevik K, Riise T, Casetta I, et al. Sun exposure and multiple sclerosis risk in Norway and Italy: The EnvIMS study. Mult Scler. 2014;20(8):1042-1049.

18. Kampman MT, Wilsgaard T, Mellgren SI. Outdoor activities and diet in childhood and adolescence relate to MS risk above the Arctic Circle. J Neurol. 2007;254(4):471-477.

19. Sintzel MB, Rametta M, Reder AT. Vitamin D and Multiple Sclerosis: A Comprehensive Review. Neurol Ther. 2018;7(1):59-85.

20. Maroney M, Hunter SF. Implications for multiple sclerosis in the era of the Affordable Care Act: a clinical overview. Am J Manag Care. 2014;20(11 Suppl): S220-S227.

21. Waubant E. Improving outcomes in multiple sclerosis through early diagnosis and effective management. Prim Care Companion CNS Disord. 2012;14(5):PCC.11016co2cc. 